\title{
Erratum to: The Handbook of Contemporary Indigenous Architecture
}

\author{
Elizabeth Grant, Kelly Greenop, Albert L. Refiti and Daniel J. Glenn
}

\section{Erratum to:}

E. Grant et al. (eds.), The Handbook of Contemporary Indigenous Architecture, https://doi.org/10.1007/978-981-106904-8

The original version of the book was inadvertently published without following consistency in Authors' biographies of Chapters 1, 3, 5, 13, 19, 20, 25, 32 and FM, which have now been updated.

\footnotetext{
The updated online version of these chapters can be found at https://doi.org/10.1007/978-981-10-6904-8_1 https://doi.org/10.1007/978-981-10-6904-8_3 https://doi.org/10.1007/978-981-10-6904-8_5 https://doi.org/10.1007/978-981-10-6904-8_13 https://doi.org/10.1007/978-981-10-6904-8_19 https://doi.org/10.1007/978-981-10-6904-8_20 https://doi.org/10.1007/978-981-10-6904-8_25 https://doi.org/10.1007/978-981-10-6904-8_32 https://doi.org/10.1007/978-981-10-6904-8 